\title{
Effects of the PAR-1 Antagonist Vorapaxar on Platelet Activation and Coagulation Biomarkers in Patients with Stable Coronary Artery Disease
}

\author{
Renske H. Olie ${ }^{1,2}$ Paola E. J. van der Meijden ${ }^{1,2}$ Henri M. H. Spronk ${ }^{1}$ Rene van Oerle ${ }^{1}$ Stale Barvik $^{3}$ \\ Vernon V. S. Bonarjee ${ }^{3}$ Hugo ten Cate ${ }^{1,2}$ Dennis W. T. Nilsen ${ }^{3}$
}

\footnotetext{
${ }^{1}$ Laboratory for Clinical Thrombosis and Hemostasis, Cardiovascular Research Institute Maastricht (CARIM), Maastricht University, The Netherlands

2 Thrombosis Expertise Center, Maastricht University Medical Center+ (MUMC+), Maastricht, The Netherlands

${ }^{3}$ Department of Cardiology, Stavanger University Hospital, Stavanger, Norway
}

TH Open 2019;3:e259-e262.
Address for correspondence Renske H. Olie, MD, Department of Internal Medicine/Thrombosis Expertise Center, Maastricht University Medical Center+, PO Box 5800, 6202 AZ Maastricht, The Netherlands (e-mail: Renske.olie@mumc.nl).

patients gave written informed consent. Patients with a previous history of myocardial infarction were randomly assigned to receive either vorapaxar ( $2.5 \mathrm{mg}$ daily) or placebo in a blinded fashion, on top of standard antiplatelet agents, as managed by the treating physicians according to standards of care. ${ }^{1,7}$ Patients using anticoagulant medication during follow-up were excluded.

A total of 135 patients with stable CAD were randomized to vorapaxar $(n=73)$ or placebo $(n=62)$. Baseline characteristics including age, comorbidity, and co-medication were well balanced between both groups ( - Table 1 ). To study the effects of long-term treatment, blood samples were taken after a mean study drug exposure of $904( \pm 149)$ days. The use of concomitant antiplatelet agents was comparable between both groups; at the time of blood sampling, 92.6 and $22.2 \%$ were treated with aspirin and clopidogrel, respectively.

According to soluble P-selectin levels, platelet activation was reduced in vorapaxar-treated patients compared with the placebo group (24.9 $\mathrm{ng} / \mathrm{mL}$ [interquartile range, IQR: $20.0-$ 31.5 ] vs. 29.3 [IQR: 22.3-32.5]; $p=0.027$ ). No difference was found in FIXa-AT levels ( $94.0 \pm 27.1$ vs. $94.5 \pm 34.0 ; p=0.93)$, FXa-AT levels (282.0 \pm 57.4 vs. $296.4 \pm 54.6 ; p=0.14)$, and TAT levels (4.0 [IQR: $3.1-5.0$ ] vs. 3.8 [IQR: $3.0-4.9] ; p=0.71$ ) between the vorapaxar-group and placebo-group (-Fig. 1). No differences in platelet or coagulation biomarker levels were found when comparing patients on single or dual antiplatelet therapy for both treatment groups ( - Appendix A).

Our data suggest that, on top of aspirin and/or clopidogrel, vorapaxar further reduces platelet activation in patients with stable CAD. This fits with the observed reduction in
License terms

Stuttgart · New York

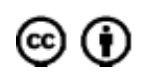

June 3, 2019

accepted

July 5, 2019
DOI https://doi.org/

10.1055/s-0039-1695710. ISSN 2512-9465. 
Table 1 Patient characteristics

\begin{tabular}{|c|c|c|c|}
\hline & $\begin{array}{l}\text { Vorapaxar }(n=73) \\
\text { mean } \pm \text { SD, or } \\
n(\%)\end{array}$ & $\begin{array}{l}\text { Placebo }(n=62) \\
\text { mean } \pm \text { SD, or } \\
n(\%)\end{array}$ & $p$-Value \\
\hline \multicolumn{4}{|l|}{ Patient characteristics } \\
\hline Age $(y)$ & $61.3 \pm 11.8$ & $60.0 \pm 10.3$ & 0.48 \\
\hline Male & $58(79.5)$ & $53(85.5)$ & 0.36 \\
\hline BMI $\left(\mathrm{kg} / \mathrm{m}^{2}\right)$ & $27.6 \pm 4.6$ & $27.7 \pm 3.7$ & 0.85 \\
\hline eGFR (mL/min) & $92.8 \pm 29.1$ & $94.1 \pm 22.7$ & 0.78 \\
\hline Diabetes mellitus & $7(9.6)$ & $4(6.5)$ & 0.51 \\
\hline Hypertension & $31(42.5)$ & $19(30.6)$ & 0.16 \\
\hline Hyperlipidemia & $32(43.8)$ & $20(32.3)$ & 0.17 \\
\hline Treatment exposure (d) & $898.5 \pm 155.1$ & $910.6 \pm 143.0$ & 0.64 \\
\hline \multicolumn{4}{|l|}{ Co-medication at sampling } \\
\hline Aspirin & $67(91.8)$ & $58(93.5)$ & 1.00 \\
\hline Clopidogrel & $18(24.7)$ & $12(19.4)$ & 0.53 \\
\hline Dual antiplatelet therapy & $17(23.3)$ & $11(17.7)$ & 0.39 \\
\hline \multicolumn{4}{|l|}{ Laboratory characteristics } \\
\hline Hemoglobin $(\mathrm{g} / \mathrm{dL})$ & $14.0 \pm 1.1$ & $14.3 \pm 1.2$ & 0.10 \\
\hline Platelet count $\left(10^{9} / \mathrm{L}\right)$ & $223.9 \pm 53.3$ & $225.6 \pm 57.1$ & 0.86 \\
\hline MPV (fL) & $10.6 \pm 0.9$ & $10.9 \pm 0.8$ & 0.18 \\
\hline
\end{tabular}

Abbreviations: BMI, body mass index; eGFR, estimated glomerular filtration rate; MPV, mean platelet volume.

Note: Values expressed as either mean \pm standard deviation (SD) or counts and percentages. Continuous variables were compared using Student's $t$ test. Categorical variables were compared using the $x^{2}$-test or Fisher's exact test when frequencies were $<5$.

atherothrombotic events in these patients in the TRA2 ${ }^{\circ}$ TIMI-50 trial. ${ }^{1}$ However, we found no additional effect on markers of coagulation activation, indicating that vorapaxar does not further reduce thrombin generation via intensified platelet inhibition.

Although P-selectin can also be secreted from endothelial cells, the increase in soluble P-selectin under (pre-)thrombotic conditions is assumed to be mainly derived from activated platelets. ${ }^{4}$ Biomarkers derived from platelets have been shown to correlate with antiplatelet therapy utilization. ${ }^{5,8,9}$ Thus, the significant reduction of soluble P-selectin levels in vorapaxartreated patients indicates a further reduction in platelet activation on top of standard antiplatelet therapy. Our study in chronic CAD patients shows that this reduction might only occur over the course of treatment, as Storey et al have previously shown that P-selectin and sCD40-ligand were comparable between vorapaxar-treated patients and control patients in the acute phase after acute coronary syndrome. ${ }^{8}$

Intrinsic tenase and prothrombinase complexes assemble on the procoagulant surface of activated platelets. Using our assays, the direct end products of these complexes, factor Xa and thrombin, were determined. If vorapaxar indeed reduces thrombin generation via intensified platelet inhibition, lower factor activity levels can be expected.

The finding that no difference is observed in factor IXa-AT, factor Xa-AT, and TAT complexes between the vorapaxar and placebo groups might be explained by the concomitant use of aspirin and clopidogrel, which already reduces platelet-depen- dent thrombin generation to such an extent that the impact of adding vorapaxar is only small. For clopidogrel, several studies have already shown an inhibitory effect on surface-generated thrombin and thrombin-induced clot formation. ${ }^{10,11}$ Furthermore, in this study samples were taken from stable CAD patients, while the beneficial effect of vorapaxar in reducing thrombin generation might become more pronounced under conditions of acute plaque rupture, when levels of thrombin rise explosively. This is in line with the consistent reduction in the rate of type 1 (spontaneous) myocardial infarction in vorapaxar-treated patients. ${ }^{1}$ Thrombin levels under stable conditions might be just too low to show a detectable reduction via platelet inhibition.

Finally, it is possible that vorapaxar has indeed no interference with the coagulation process, in accordance with the absence of effect on parameters of thromboelastography in a previous study. ${ }^{12}$ Theoretically, part of the beneficial effect of vorapaxar on the risk of ischemic events might also be attributable to effects of vorapaxar on the vascular endothelium. Since PAR-1 on endothelial cells and vascular smooth muscle cells mediates mitogenic effects, vorapaxar might be effective in reducing vascular remodeling and consecutive progression of atherosclerosis.

Strengths of our analysis include the simultaneous measurement of both coagulation and platelet biomarkers in a patient population on long-term treatment with stable CAD. Limitations are the relatively small sample size, the fact that we were only able to measure at a single time point, and that total 

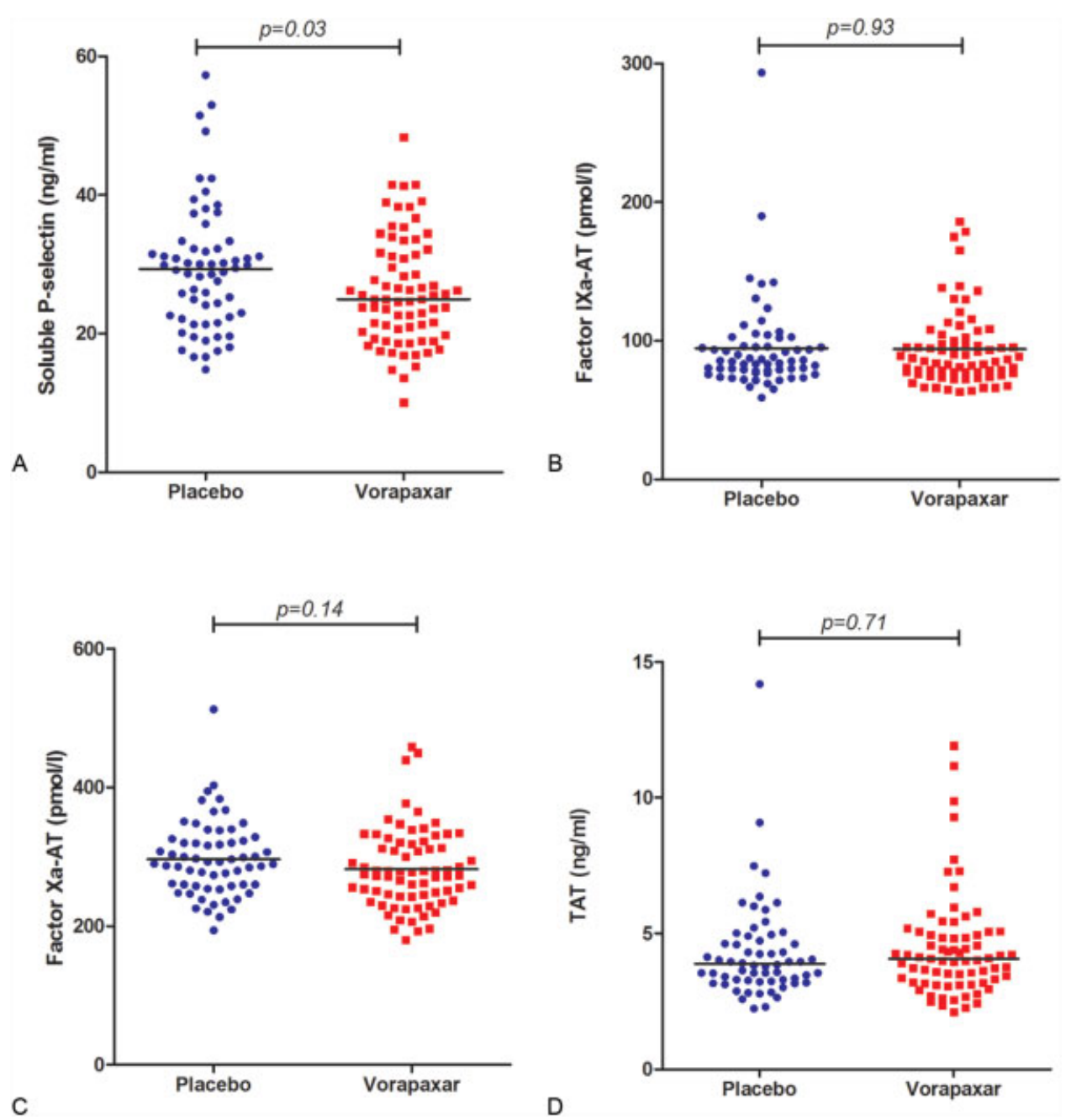

Fig. 1 Results of soluble P-selectin, FIXa-AT, FXa-AT, and TAT complexes in vorapaxar-treated patients and placebo group. Compared with the placebo group, vorapaxar-treated patients had significantly lower levels of soluble P-selectin $(p=0.03)$ (A). No significant differences were found in factor IXa-antithrombin (FIXa-AT) (B), factor Xa-antithrombin (FXa-AT) (C), and thrombin-antithrombin (TAT) (D) complexes between vorapaxar-treated patients and the placebo group.

platelet P-selectin was not assessed. Although we cannot fully prove that the detected decrease in soluble P-selectin is related to additional platelet inhibition, it is likely to be at least in part a reflection of total platelet P-selectin. ${ }^{4}$

In conclusion, our data suggest that the beneficial effect of vorapaxar on top of standard antiplatelet therapy in stable CAD patients is likely due to further attenuation of platelet reactivity, without detectable reduction in thrombin generation.

\section{Funding}

The logistics of this study (costs related to preparation and shipping of samples) were financially supported by MSD (Schering-Plough), Norway. There was no involvement of MSD in the conduct of the study, in the collection, data analysis and interpretation of the data, and in preparation, review, or approval of the manuscript.

\section{Conflict of Interest}

Dr. Olie reports nonfinancial support and other from MSD (Schering-Plough), Norway, during the conduct of the study. Dr. Nilsen reports grants from MSD (Schering-
Plough), Norway, during the conduct of the study. All other authors reported no conflict of interest.

\section{References}

1 Morrow DA, Braunwald E, Bonaca MP, et al; TRA 2P-TIMI 50 Steering Committee and Investigators. Vorapaxar in the secondary prevention of atherothrombotic events. N Engl J Med 2012;366(15): 1404-1413

2 Chackalamannil S, Xia Y, Greenlee WJ, et al. Discovery of potent orally active thrombin receptor (protease activated receptor 1 ) antagonists as novel antithrombotic agents. J Med Chem 2005;48 (19):5884-5887

3 Chackalamannil S. Thrombin receptor (protease activated receptor-1) antagonists as potent antithrombotic agents with strong antiplatelet effects. J Med Chem 2006;49(18):5389-5403

4 Blann AD, Nadar SK, Lip GY. The adhesion molecule P-selectin and cardiovascular disease. Eur Heart J 2003;24(24):2166-2179

5 Thomas MR, Storey RF. Effect of P2Y12 inhibitors on inflammation and immunity. Thromb Haemost 2015;114(03):490-497

6 Xiao Z, Théroux P. Clopidogrel inhibits platelet-leukocyte interactions and thrombin receptor agonist peptide-induced platelet activation in patients with an acute coronary syndrome. J Am Coll Cardiol 2004;43(11):1982-1988 
7 Morrow DA, Scirica BM, Fox KA, et al. Evaluation of a novel antiplatelet agent for secondary prevention in patients with a history of atherosclerotic disease: design and rationale for the Thrombin-Receptor Antagonist in Secondary Prevention of Atherothrombotic Ischemic Events (TRA 2 degrees P)-TIMI 50 trial. Am Heart J 2009;158(03):335.e3-341.e3

8 Storey RF, Kotha J, Smyth SS, et al. Effects of vorapaxar on platelet reactivity and biomarker expression in non-ST-elevation acute coronary syndromes. The TRACER Pharmacodynamic Substudy. Thromb Haemost 2014;111(05):883-891

9 Steinhubl SR, Badimon JJ, Bhatt DL, Herbert JM, Lüscher TF. Clinical evidence for anti-inflammatory effects of antiplatelet therapy in patients with atherothrombotic disease. Vasc Med 2007;12(02):113-122
10 Gurbel PA, Bliden KP, Guyer K, Aggarwal N, Tantry US. Delayed thrombin-induced platelet-fibrin clot generation by clopidogrel: a new dose-related effect demonstrated by thrombelastography in patients undergoing coronary artery stenting. Thromb Res 2007;119(05):563-570

11 van der Meijden PE, Feijge MA, Giesen PL, Huijberts M, van Raak LP, Heemskerk JW. Platelet P2Y12 receptors enhance signalling towards procoagulant activity and thrombin generation. A study with healthy subjects and patients at thrombotic risk. Thromb Haemost 2005;93(06):1128-1136

12 Gurbel PA, Bliden K, Bell R, et al. Vorapaxar pharmacodynamics: effects on thrombogenicity, endothelial function, and interactions with clopidogrel and aspirin. J Am Coll Cardiol 2018;71 (11, Suppl):A76

Appendix A Comparison of platelet and coagulation biomarkers in patients with single versus dual antiplatelet therapy

\begin{tabular}{|l|l|l|l|l|}
\hline Treatment group & Biomarker & SAPT & DAPT & $p$-Value \\
\hline \multirow{5}{*}{ Vorapaxar } & P-selectin & $26.6 \pm 8.0$ & $24.6 \pm 6.8$ & 0.36 \\
\cline { 2 - 5 } & FIXa-AT & $94.4 \pm 25.5$ & $88.4 \pm 28.6$ & 0.42 \\
\cline { 2 - 5 } & FXa-AT & $285.4 \pm 62.1$ & $265.2 \pm 41.2$ & 0.21 \\
\cline { 2 - 5 } & TAT & $4.5 \pm 2.1$ & $4.0 \pm 1.3$ & 0.41 \\
\hline \multirow{5}{*}{ Placebo } & P-selectin & $28.9 \pm 8.4$ & $31.7 \pm 12.1$ & 0.37 \\
\cline { 2 - 5 } & FIXa-AT & $96.6 \pm 36.9$ & $87.2 \pm 19.3$ & 0.54 \\
\cline { 2 - 5 } & FXa-AT & $297.4 \pm 56.4$ & $286.0 \pm 47.8$ & $3.5 \pm 1.3$ \\
\cline { 2 - 5 } & TAT & $5.2 \pm 2.9$ & 0.26 \\
\hline
\end{tabular}

Abbreviations: DAPT, dual antiplatelet therapy; FIXa-AT, factor IXa-antithrombin; FXa-AT, factor Xa-antithrombin; SAPT, single antiplatelet therapy; TAT, thrombin-antithrombin. 